\title{
Progress report on the K2 RR Lyrae survey
}

\author{
Róbert Szabó ${ }^{1, \star}$ \\ ${ }^{1}$ Research Centre for Astronomy and Earth Sciences, Konkoly Observatory, H-1121, Budapest, Konkoly \\ Thege Miklós út 15-17., Hungary
}

\begin{abstract}
We have initiated a large survey with K2, to observe thousands of RR Lyrae stars along the ecliptic. The high photometric precision and the 70-90-day continuous coverage will allow us to investigate the light variation of these galactic structure tracer variable stars with unprecedented detail. The survey will help us to conduct a thorough statistical study of RR Lyrae pulsation dynamics including old and recently discovered dynamical phenomena, like resonances, non-radial modes, period-doubling and the Blazhko effect. In this contribution I present a status report of the survey.
\end{abstract}

\section{Introduction}

With the failure of the second reaction wheel of Kepler, a new mission, dubbed K2, was conceived ([4]). The new mission observes ecliptic fields for 70-90 days with a precision which is comparable to the original mission. $\mathrm{K} 2$ is a bona fide community endeavour: without a central, pre-determined research plan, exoplanets, stellar (asteroseismic and other variable) sources, extragalactic objects, microlensing events, and even Solar System objects have been proposed by community members. ${ }^{1}$ This opened up new possibilities in RR Lyrae research, too, since compared to the original Kepler field, where roughly 50 RR Lyrae stars were found ([1, 6, 7]), thousands of RR Lyrae stars can be observed with K2. Members of the Kepler Asteroseismic Science Consortium (KASC) RR Lyrae and Cepheids Working Group initiated a large survey to ensure that as many RR Lyrae stars will be observed with $\mathrm{K} 2$ as possible. ${ }^{2}$ This contribution discusses the present status of this undertaking.

\section{The K2 RR Lyrae survey}

As Figure 1 demonstrates, our group was successful in proposing RR Lyrae targets throughout the whole K2 Mission. From Campaign 4 the proposals are submitted through the NASA NSPIRES system. ${ }^{3}$ Figure 1 also shows that RR Lyrae stars are measured in very different environments: in the Galactic halo and halo sub-structures (Sagittarius-stream), in the Galactic bulge, in globular clusters (M4, M80), and even in external galaxies (e.g., the Leo IV dwarf spheroidal galaxy; [5]). We used all possible variable star catalogs for target selection, and heavily relied on the Catalina Sky Survey

\footnotetext{
^szabo.robert@csfk.mta.hu

${ }^{1}$ https://keplerscience.arc.nasa.gov/k2-approved-programs.html

${ }^{2}$ The same strategy applies to Cepheids, both classical and Type II ones (see Molnár et al., these proceedings).

${ }^{3}$ https://nspires.nasaprs.com/
} 


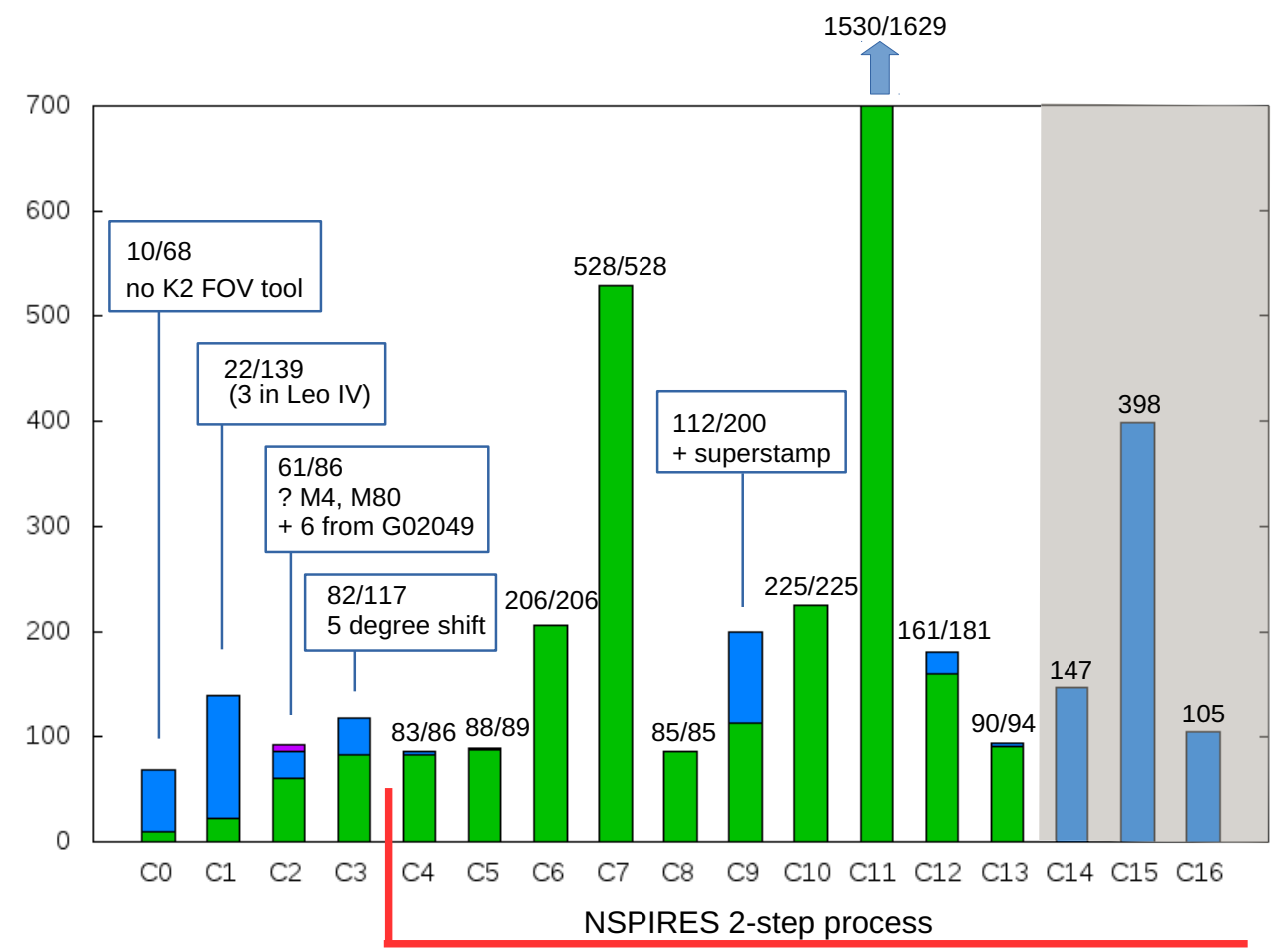

Figure 1. Statistics of the number of proposed (blue) and observed (green) RR Lyrae targets in the K2 campaigns. The K2 RR Lyrae survey has been largely successful in securing most of the known RR Lyrae stars for K2 observations. The grey area shows those campaigns that are scheduled at the time of writing. Two more campaigns $(\mathrm{C} 17, \mathrm{C} 18)$ are envisaged, contingent on available fuel on board the Kepler spacecraft.

results ([3]). A more detailed description of the target selection process can be found in Plachy et al. (2016, [8]).

By the end of the K2 Mission we expect to have light curves of over 3000 RR Lyrae stars in the Kepler photometric band (Kp). The majority of the targets is observed with long cadence (30min sampling), while a small fraction is observed with short cadence (1-min sampling) mode. The distribution of the K2 RR Lyrae stars on the sky is plotted in Figure 2. As correction maneouvres are necessary to keep the spacecraft on-target, special treatment of the light curves (in particular for highamplitude variables) is needed. Hence, we developed the Extended Aperture Photometry method ([9]) that delivers optimal results for our targets. This way quasi-continuous light curves covering $70-90$ days are derived with high photometric precision, which makes the K2 RR Lyrae survey unique. Our project nicely complements other sky surveys, e.g. OGLE, Gaia or LSST.

The main scientific motivation behind our efforts is a statistical study of the occurrence of dynamical phenomena (Blazhko effect, period doubling, nonradial modes, etc.) in RR Lyrae stars as a function of age, metallicity, and Galactic position. By these investigations we hope to achieve a better 


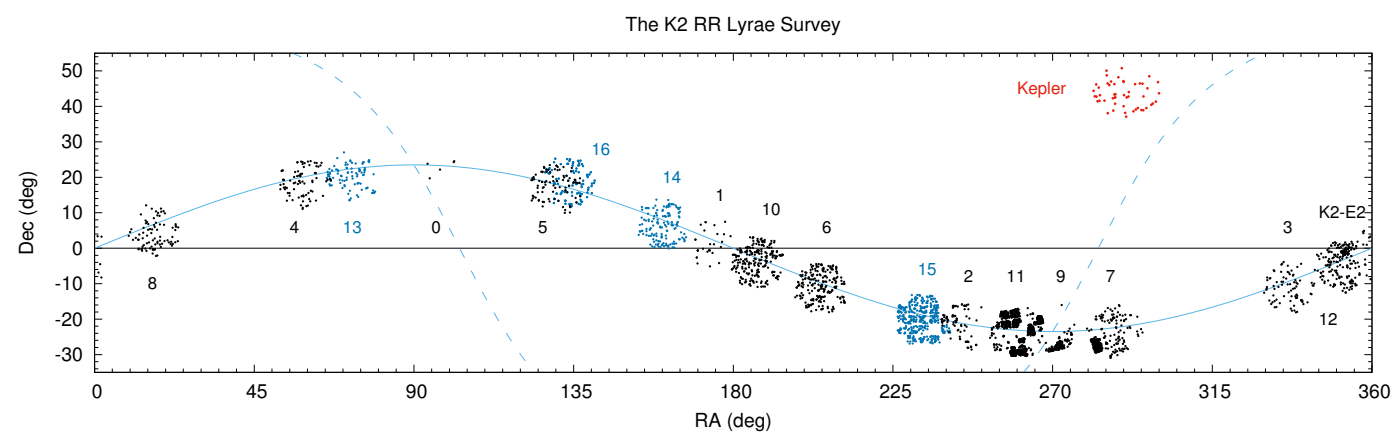

Figure 2. The footprint of the K2 RR Lyrae survey along the Ecliptic. Approved (blue) and observed (black) RR Lyrae targets are marked in the K2 campaign fields. The original Kepler field is also plotted for reference (red).

understanding of recently discovered (period doubling, [10]; extra frequencies, [11]) or century-old (like the Blazhko-modulation, [2]) dynamical phenomena occurring in RR Lyrae stars.

\section{Summary}

We presented the K2 RR Lyrae survey which will be a treasure trove for pulsation and dynamical studies for many years to come. It contains representatives of all currently known subtypes (RRab, $\mathrm{RRc}, \mathrm{RRd}$, modulated and non-modulated variants, as well). The corrected, "ready-to-use" RR Lyrae light curves from survey will be made publicly available (Molnár et al. in prep.).

Acknowledgments: This project has been supported by the Hungarian National Research, Development and Innovation Office - NKFIH K-115709, the GINOP-2.3.2-15-2016-00003 grant, and the LP2014-17 Lendület grant of the Hungarian Academy of Sciences. This work includes data collected by the K2 mission. Funding for the K2 mission is provided by the NASA Science Mission Directorate. We acknowledge the Kepler team and engineers for their efforts to keep this fantastic instrument alive and design a new mission.

\section{References}

[1] Benkő, J., Plachy, E., Szabó, R., Molnár, L., \& Kolláth, Z., ApJS, 213, 31 (2014)

[2] Blazhko, S., AN, 175, 325 (1907)

[3] Drake, A. J., Graham, M. J., Djorgovski, S. G., et al., ApJS, 213, 9 (2014)

[4] Howell, S., Sobeck, C., Haas, M. et al., PASP, 126, 398 (2014)

[5] Molnár, L., Pál, A., Plachy, E. et al., ApJ, 821, 2 (2014)

[6] Moskalik, P., Smolec, R., Kolenberg, K. et al., MNRAS, 447, 2348 (2015)

[7] Nemec, J. M., Smolec, R., Benkő, J. M. et al. MNRAS, 417, 1022 (2011)

[8] Plachy, E., Molnár, L., Szabó, R., Kolenberg, K., \& Bányai, E., Communications from the Konkoly Observatory, 105, 19 (2016)

[9] Plachy, E., Klagyivik, P., Molnár, L., Sódor, Á., Szabó, R., in Seismology of the Sun and the

Distant Stars, EPJ Web of Conferences, in press (2017)

[10] Szabó, R., Kolláth, Z., Molnár, L. et al. MNRAS, 409, 1244 (2010)

[11] Szabó, R., Benkő, J., Paparó, M. et al. A\&A, 570, A100 (2014) 